\author{
Jerzy Brzozowski iD \\ Université Jagellonne de Cracovie \\ jerzy.brzozowski@uj.edu.pl
}

\title{
Représentation de la réalité et la post-vérité en traduction
}

L'éminent linguiste américain Dwight Bollinger, cité par Maria Tymoczko, avait remarqué en 1977 déjà que la tendance de notre esprit est de négliger la différence, lorsqu'il s'agit de deux objets qui se ressemblent nettement En principe, il faut que la différence saute aux yeux pour que nous concédions qu'en effet, la différence existe ${ }^{1}$. Et c'est un mécanisme qui vaut tout autant pour la traduction, affirme Maria Tymoczko [2004:30-31].

C'est un mécanisme que je voudrais étudier sur quelques exemples de traduction récents. Il ne s'agit pas forcément de mauvaises traductions, comme le titre de cet article pourrait le laisser suggérer ; certaines d'entre elles sont même de jolies trouvailles. Mais le dénominateur commun de ces exemples est qu'elles nous montrent des manipulations

« Always one's first impulse, on encountering two highly similar things, is to ignore their differences in order to get them into a system of relationships where they can be stored, retrieved and otherwise made manageable. The sin consists in stopping there. And also in creating an apparatus of absolute equality and absolute inequality, and uses the latter only when the unlikeness that it represents is so gross that it bowls you over » [Bollinger, 1977 : 5] 
absolument conscientes de l'original. Ce qui est donc en question n'est pas tellement d'analyser la qualité de la traduction, le degré de fidélité de celle-ci, son acceptabilité linguistique : non, il s'agit plutôt de nous mettre en examen nous-mêmes, les lecteurs, et notre degré de complaisance avec une manipulation, d'acceptabilité de ce qui n'est pas vrai.

Car, nous dit-on, nous vivons dans une époque de post-vérité :

After much discussion, debate, and research, the Oxford Dictionaries Word of the Year 2016 is post-truth - an adjective defined as 'relating to or denoting circumstances in which objective facts are less influential in shaping public opinion than appeals to emotion and personal belief ${ }^{2}$.

Pour arriver aux situations où l'on admet que « les faits objectifs ont moins d'influence dans le discours public que les émotions et croyances personnelles ", il fallait certainement un processus assez long; probablement l'un des premiers à remarquer jusqu'à quel point la vérité à notre époque cause problème était l'écrivain et journaliste américain Ralph Keyes qui en 2004 déjà avait publié son best-seller The Post-Truth Era : Dishonesty and Deception in Contemporary Life où nous pouvons lire quelques phrases brillantes que je me permets de citer [Keyes, 2004 : 13-16] :

At one time we had truth and lies. Now we have truth, lies, and statements that may not be true but we consider too benign to call false. [...] Dishonesty inspires more euphemisms than copulation or defecation. [...] We're "economical with the truth," we "sweeten it," or tell "the truth improved." [...] At worst we admit to "misspeaking," or "exercising poor judgment." Nor do we want to accuse others of lying. We say they're in denial. A liar is "ethically challenged," someone for whom "the truth is temporarily unavailable."

This is post-truth. In the post-truth era, borders blur between truth and lies, honesty and dishonesty, fiction and nonfiction. Deceiving others becomes a challenge, a game, and ultimately a habit.

La vérité est donc devenue un défi, parler de la vérité est désormais un défi. C'est pourtant, nous a-on enseigné, la denrée que le traducteur est censé fournir, il est payé juste pour cela : « Le traducteur est producteur des équivalences, un professionnel dans la communication travaillant

2 Source : https://en.oxforddictionaries.com/word-of-the-year/word-of-the-year-2016, consultée le 08 octobre 2017. 
pour les gens qui payent pour le croire ; pour croire qu'à chaque niveau où c'est relevant, $\mathrm{B}$ est équivalent à $\mathrm{A} »[\mathrm{Pym}, 2007$ : XX, trad. J.B.]. Sa tâche principale est donc de représenter la vérité d'un texte, parlé ou écrit. Mais à l'époque de la post-vérité il est peut-être excusable d'être " économe dans la représentation de la vérité ", de représenter une "vérité améliorée », sans qu'on se sente « défié éthiquement »?

Je me permettrai donc d'examiner quelques exemples annoncés cidessus, en commençant par des cas plus ou moins anodins. Mais graduellement, ces cas se feront moins anodins, et finalement je crois pouvoir fournir des exemples capables d'ébranler les certitudes confortablement établies - car, je le répète après Dwight Bollinger, notre première réaction instinctive vis-à-vis des deux objets qui se ressemblent plus ou moins est de négliger les différences, et il nous faut quasiment un choc pour admettre que les limites de la ressemblance (ou tolérance au laxisme généralisé de l'époque du post-vrai) ont été franchis.

Voici mon premier exemple : Alice au pays des merveilles, et la citation que voici : « Mais alors, dit Alice, si le monde n'a absolument aucun sens, qu'est-ce qui nous empêche d'en inventer un ? » La citation a paru quelque peu bizarre à Clémentine Beauvais qui s'est donné la peine de la vérifier, dans toutes les éditions accessibles, aussi bien dans la version papier comme numérique. Le résultat de cette recherche est étonnant : cette citation n'existe pas, sauf sur Internet. Voici ses conclusions :

- La citation est probablement en français à l'origine ;

- Elle date de 2000 ou 2001;

- Elle pourrait mixer une citation du livre avec la chanson d'introduction du film Disney.

L'auteure termine avec une anecdote :

Ayant fait remarquer à certaines personnes sur Facebook que cette phrase n'existait sans doute pas, j'ai eu le commentaire suivant :

- Mais alors, si la citation n'existe absolument pas, qu'est-ce qui nous empêche de l'inventer?

La riposte est jolie. Mais à mon avis, Carroll ne l'aurait pas likée... ${ }^{3}$

On dira que c'est une petite post-vérité sans importance, et qu'elle ne fait tort à personne : c'est ce que pensent probablement plusieurs

3 Source : http://www.madmoizelle.com/alice-pays-merveilles-citation-fausse-783539, consultée le 15 octobre 2017. 
internautes, comme celui cité ci-dessus par Mme. Beauvais. Le problème supplémentaire est que cette citation prétendue est d'une platitude désolante, et sans le dessin qui l'accompagne, il serait quasi impossible d'y prêter l'attention. Une personne avide de popularité s'est emparée d'un nom célèbre pour séduire quelques ingénus, au dépens de Lewis Carroll.

Deuxième exemple : L'auteur de La caverna de las ideas a une assistante bénévole qui explique les difficultés de la eidesis : sa traductrice anglaise, comme le constate Yvonne Lindquist dans son article « Manipulating the matricial norm » de 2010. Voici les fragments de la traduction française et anglaise du texte de Carlos Somoza :

Il est surprenant que, dans son édition érudite de l'original, Montalo ne fasse même pas référence à la forte eidesis que révèle le texte, du moins tout au long de ce premier chapitre. Mais il est également possible qu'il ignore l'existence d'un procédé littéraire aussi étonnant. A titre d'exemple pour l'édification du lecteur curieux et aussi pour expliquer avec sincérité comment j'en suis venu à découvrir l'image cachée dans ce chapitre [...] [Somoza, 2003, apud Lindquist 2010 : 77]

I find it surprising that, in his scholarly edition of the original, Montalo should make no mention of the powerful eidesis present in the text, at least throughout the first chapter. But maybe he didn't know about this strange literary device. It's not unusual to find translators, even among the most erudite, who are not familiar with a literary technique which may, in any case, have been used by only a handful of Greek writers - in some ways the most celebrated ones - and whose main feature is precisely that it is only noticed by those who know about it. By way of example for the curious reader, and also to be honest about how I came to discover the image hidden in this chapter $[\ldots]$

[Somoza, 2002, apud Lindquist 2010 : 78]

En fait, La caverna de las ideas de Carlos Somoza est un polar dont le narrateur est prétendument le traducteur du grec vers l'espagnol ; l'histoire racontée dans le texte soi-disant traduit envahit la vie du soi-disant traducteur... Dans ce jeu ingénieux, une chose est claire : l'auteur tient à ce que le fictif se mêle au réel, autrement dit, sa fiction, mensongère par essence, devient plus ouvertement mensongère encore. Soit : l'auteur a tous les droits. Mais le traducteur? Le traducteur réel, et non le narrateur-traducteur fictif du roman? Les traducteurs réels, français et suédois 
font tout simplement ce qui revient au traducteur, ils traduisent. Toutefois la traductrice vers l'anglais, Sonia Soto, sort de son rôle, elle entre dans le rôle de l'auteur et ajoute ses propres commentaires, sans le déclarer (le texte souligné, voir ci-dessus). Pourquoi le fait-elle ? Yvonne Lindqvist, qui analyse ces exemples dans l'article cité, se contente d'opiner que c'est le manque de respect pour l'original, son objectif majeur étant d'analyser son corpus dans la perspective des polysytstèmes. Pour ma part, je vois ici une certaine affinité avec les prétentions mégalomanes de l'internaute anonyme qui cherche la gloriole en forgeant un faux adage au dépens d'un auteur célèbre. Ici, la traductrice entre dans les compétences de l'auteur, en ajoutant quelques grains de sel (attique, pense-t-elle probablement) censés affiner le goût du roman de divertissement - on peut donc conclure que c'est un cas classique d' " ennoblissement » Bermanien [Berman, 1985], poussé, il est vrai, assez loin d'un point de vue quantitatif.

Tel n'est pas le cas de mon troisième exemple que j'emprunte aux deux articles de Patrick Zabalbeascoa (Barcelone), le premier paru dans le Volume 2 du Translator de 1996 et repris ensuite avec des changements majeurs dans une publication collective de 2012. Il s'agit d'une série télévisée Fawlty Towers de John Cleese qui joue le rôle principal du propriétaire d'un hôtel prétendument " chic ». Il est aidé par un domestique stupide et gauche qui ne parle presque pas anglais. Or il se trouve que le grotesque Manuel est Espagnol : un problème, si l'on voulait diffuser cette série en Espagne. Il est clair que les Espagnols n'allaient pas aimer ce personnage, et conséquemment le sort de toute la série était menacé : pourtant, comme le déclare Patrick Zabalbeascoa, ce fut un succès, avec le droit à plusieurs rediffusions [Zabalbeascoa, 1996 : 240]. Comment cela a-t-il été possible ? Eh bien, grâce à l'astuce suivante :

Fawlty Towers a été difusée en Espagne en trois langues. [...] Dans la version doublée en espagnol, Manuel-de-Barcelone est devenu Paolo-de-Napoli. [...] Dans la version doublée en espagnol, Manuel-from-Barcelona devint Manuel-de-Jalisco [Mexique, J.B.]. Seulement la version basque a gardé l'espagnol dans la traduction, comme c'était le cas dans l'original.

[Zabalbeascoa, $2012: 322-323$, trad. J.B.]

On pourrait alléguer que dans le doublage, cette astuce était inévitable. Or, elle n'en était pas : on pouvait aussi se servir d'un dialecte provincial, faire du pauvre Manuel un paysan arriéré de Sierra Nevada ou 
des communes frontalières de Estremadura. Toujours est-il que le public espagnol ne l'aurait pas probablement apprécié ; il s'agit donc d'une décision politique, imposée par le distributeur, ce que Patrick Zabalbeascoa admet ouvertement :

It is frequently the case that translators do not have the last word on their work, unlike many other professionals. The example from FT, where a character's birthplace was changed from Barcelona all the way to Mexico was not the result of a decision taken by the translator. [...] For such radical departures from the original the translator needs permission - or imposition - from someone of greater authority. In the context of dubbing [...] the translators are usually among the poorest paid and leas kindly regarded.

[Zabalbeascoa, 1996 : 249]

Il faut donc se rendre à l'évidence que le traducteur n'a pas toujours le dernier mot, et se voit imposer des solutions qu'il n'aurait peut-être pas choisies... ou encore, il n'attend pas qu'on les lui impose, sa conscience étant à l'unisson avec celle du distributeur, propriétaire du journal, le pouvoir politique en place, etc. On peut croire que cette dernière situation a eu lieu dans le quatrième exemple que m'a fourni l'article de Lance Hewson, «Les images du lecteur », paru dans les Palimpsestes en 1995 (donc plus ou moins à la même époque de la publication de l'article de Zabalbeascoa):

NATO must make putting an end to the hostilities its number one priority over the next few months - at whatever cost.

Contribuer à l'arrêt des hostilités en Yougoslavie en utilisant tous les moyens possibles, l'OTAN en particulier, devrait être le devoir numéro un de l'Europe dans les prochains mois.

Le texte anglais et sa traduction française ont été publiés en 1993 dans un magazine bilingue franco-anglais Eurodiagnostic, destiné aux étudiants. Ceux qui ne parlent pas anglais reçoivent toutefois dans la traduction française un message tout à fait différent : dans le texte anglais le sujet est NATO, et le verbe « must » est renforcé par la locution adverbiale at whatever cost, « à tout prix »-il s'agit donc d'une responsabilité à prendre inévitablement, sans tergiverser. Dans le texte français apparait le verbe au conditionnel, « devrait... », «à tout prix » devient « en utilisant tous les moyens possibles », 
et le sujet... c'est l'Europe, absente de l'original, tandis que l'OTAN devient à peine " un moyen » entre tant d'autres " moyens possibles ».

Ici, la situation est très claire : nous avons une manipulation politique du message qui ne cherche pas d'alibi. Le traducteur écrit ce que le public français à l'époque - ou du moins les autorités françaises de l'époque voudraient entendre, en distordant, ou plutôt - soyons honnêtes : en faussant le message. Il est toujours possible que le traducteur exprime ses propres convictions dans ce qu'il écrit. Il est également possible que, comme dans le cas précédent, c'est quelqu'un de plus haut placé qui lui a " suggéré » l'abandon radical du sens de l'original. Peu importe, en somme : nous avons quitté le champ du jeu, du non-sérieux, même si dans le show business on gagne de l'argent tout à fait sérieux. Dans le cas présent, c'est l'honnêteté du discours public qui est en cause, et l'enjeu éthique de nos engagements politiques : devrions-nous contribuer, dans la mesure du possible, à mettre fin aux hostilités, ou, au contraire, devonsnous le faire à tout prix ?

Mes $5^{\mathrm{e}}$ et $6^{\mathrm{e}}$ exemples datent aussi de la même époque, le début des années 1990, mais curieusement, il nous mettent au cœur du débat qui secoue actuellement l'église catholique. Les traductions que je propose d'analyser témoignent d'un certain courant de pensée qui précède l'actuel remue-ménage, à savoir, il atteste les révendications féministes contre la vision univoquement machiste de la Bible. Voici les mêmes fragments dans la traduction officielle des épiscopats francophones et la traduction « inclusive » de 1994, qui s' auto-proclame comme « la première traduction égalitaire $\gg$.

Le premier exemple, provenant de l'Évangile selon saint Jean est anodin :

Les scribes et les pharisiens lui amènent une femme qu'on avait surprise en situation d'adultère.

(Bible de l'Association Épiscopale Liturgique pour les pays Francophones: AELF)

A couple had been caught in the act of adultery, though the scribes and Pharisees brought only the woman.

(The Inclusive New Testament. The First Egalitarian Translation: INT, apud Gomola 2012)

En fait, cela est vrai : la traduction « inclusive » ajoute ce que l'original cache pour des raisons, admettons, historico-culturelles. Dans le site 
consacré à la littérature religieuse, www.christianbook.com, cette traduction a été disponible en octobre 2017 encore, avec un forum des lecteurs apparemment non censuré, où l'on pouvait trouver les opinions des partisans de cette traductions (neuf opinions), mais aussi des opposants (vingt opinions), comme celle qui suit :

If you picked up this Bible and read a few random paragraphs, you might not find anything different from other translations. In places it is very readable. [...]. I don't mind that «king» has been changed to «ruler,» or that «servants» is rendered «attendants» (for no good reason). But [...] there are major problems in changing «Son of God» to "God's Own» (since «Own» can be anything, not necessarily a family relation). Most troubling of all are Paul's passages about marriage and sexuality, which are distorted so as to approve of behaviors that Paul clearly condemned. To the serious Christian, the Bible is what it is - words of comfort, yes, but also words of warning and words of command. [...] In trying to make the Bible inoffensive to «oppressed» groups, the translators have created something serious Christians cannot accept ${ }^{4}$.

L'auteur se référant aux écrits de saint Paul pense certainement aux passages comme celui-ci, provenant de l'Épître aux Colossiens $(3,18)$ :

Vous les femmes, soyez soumises à votre mari (AELF)

You who are in committed relationships, be submissive to each other (INT)

Cette dernière traduction est destinée, évidemment, à conforter les couples non-mariés et couples homosexuels. Le commentaire cité cidessus, qui la critique de façon ferme, mais non injurieuse, a un titre: An unbiblical Bible, La Bible non-biblique. Ou plutôt avait ce titre, car la traduction " inclusive " et le forum des critiques ont désormais disparu et l'adresse citée est introuvable. Apparemment, les propriétaires de cette entreprise, " the largest catalog / Internet company serving the Christian marketplace, frequently cited as «the source»for Christian products $»^{5}$, n'ont plus l'intention de propager un produit contesté par l' Eglise catholique et plusieurs Eglises protestantes des EUA, influentes et

4 Source : https://www.christianbook.com/Christian/Books/product_reviews?sku=512138, consultée le 23 octobre 2017.

5 Source : https://www.christianbook.com/page/info/company-history, consultée le 10 mai 2018. 
apparemment, en majorité, plus conservatrices que les Éuropéennes. Cette traduction est néanmoins toujours disponible sur le site Amazon.com ${ }^{6}$, avec droit de critique, bien sûr : toutes favorables à cette initiative, les plus osées déplorant les caractères d'imprimerie trop petits... Amazon.com, quant à elle, semblerait-elle censurer son forum d'une autre façon, en éliminant les opinions susceptibles de diminuer le chiffre des ventes?

On peut se demander si réellement les révendications féministes, ou même " égalitaires » sensu largo ont quelque chose à voir avec le débat actuel qui est en passe de, peut-être, diviser l'église catholique ? Et qui, bien au-delà de l'église catholique, enveniment notre discours public ? Ma réponse est affirmative, et pas du tout pour blâmer les féministes. Je tiens seulement à signaler que dans notre époque de post-vérité, quand deux positions nettement contraires se choquent, on manipule et on censure ce qu'on n'a pas envie d'entendre, dès que l'on détient un pouvoir suffisant ; mais il est désagréable de l'avouer, bien évidemment. On a toutefois une solution plus agréable : celle de plaire à la majorité, celle de pratiquer la complaisance vis-à-vis du lecteur dans des cas idéologiquement délicats ou - c'est mon dernier exemple - lorsqu'on est un traducteur assermenté (polonais en l'occurence), d'octroyer libéralement à notre client un titre universitaire contestable et ultérieurement non accordé, sans attendre la décision d'une commission universitaire compétente ${ }^{7}$.

Il est facile donc de passer, dans notre époque de post-vérité, que ce soit dans la littérature, dans les médias voire dans les documents officiels, de la vérité pure et simple, aux représentations de la vérité qui l'adoucissent, l'atténuent, ou l'humanisent, si l'on préfère un euphémisme de plus; et la traduction y joue un rôle prépondérant.

\section{Bibliographie}

Berman, A. (1985), La traduction et la lettre ou l'auberge du lointain, dans : Berman, A., Tours de Babel, Trans-Europ Repress, Paris.

Bollinger, D. (1977), Meaning and Form, Longman, New York.

6 Source : https://www.amazon.com/product-reviews/1580512135/ref=cm_cr_arp_d_ viewpnt_rgt?ie=UTF8\&filterByStar $=$ critical\&showViewpoints $=0$ \&pageNumber $=1$, consultée le 05 mai 2018.

7 L'auteur de cet article fut confronté à la situation décrite ci-dessus comme président de ladite commision, c'est pourquoi tous les détails concernant le cas concret doivent rester confidentiels. 
Gomola, A. (2012), « Od św. Hieronima do feministek - tłumacz jako vox Dei », Znak 688.

Hewson, L. (1995), « Images du lecteur », Palimpsestes, 9, https://doi. org/10.4000/palimpsestes.686.

Keyes, R. (2004), The Post-Truth Era : Dishonesty and Deception in Contemporary Life, St. Martin's Press, New York.

Lindqvist, Y. (2010), « Manipulating the Matricial Norms. A Comparison of the English, Swedish and French Translations of La caverna de las ideas by José Carlos Somoza », dans : Gile, D., Hansen, G., Pokorn N.K. (eds), Why Translation Studies Matters, Amsterdam-Philadelphia, pp. 69-82, https://doi. org/10.1075/btl.88.07lin.

Pym, A. (2007), « Natural and directional equivalence in theories of translation », Target, 19(2), https://doi.org/10.1075/target.19.2.07pym.

Priests for Equality (2007), The Inclusive Bible: The First Egalitarian Translation, Rowman and Littlefield Publishers, Lanham.

Somoza, J.C. (2003), La caverne des idées, trad. M. Millon, Actes Sud, Arles.

Somoza, J.C. (2002), The Athenian Murders, trad. S. Soto, Abacus, London.

Somoza, J.C. (2000), La caverna de las ideas, Alfaguarra, Madrid.

Tymoczko, M. (2004), Similarity and Difference in Translation, Guaraldi, Rimini. Zabalbeascoa, P. (1996), « Translating Jokes for Dubbed Television Situation Comedies », The Translator, 2(2), pp. 235-257, https://doi.org/10.1080/135 56509.1996.10798976.

Zabalbeascoa, P. (2012), « Translating Heterolingual Audiovisual Humor: Beyond the Blinkers of Traditional Thinking », dans : Muñoz-Basols, J., Fouto, C., Soler González, L., Fisher, T. (eds), The Limits of Literary Translation: Expanding Frontiers in Iberian Languages, Edition Reichenberger, Kassel.

\section{Sites internet consultés :}

http://www.madmoizelle.com/alice-pays-merveilles-citation-fausse-783539 https://en.oxforddictionaries.com/word-of-the-year/word-of-the-year-2016 https://www.aelf.org/bible (Bible de l'Association Épiscopale Liturgique pour les pays Francophones)

https://www.christianbook.com/Christian/Books/product_reviews?sku=512138 https://www.amazon.com/product-reviews/1580512135/ref=cm_cr_arp_d_ viewpnt_rgt?ie $=$ UTF $8 \&$ filterByStar $=$ critical\&showViewpoints $=0 \&$ pageNu mber $=1$ 


\section{RÉSUMÉ}

Le présent article analyse six exemples de traduction récents qui relèvent des domaines de la littérature, du film, des mass-média et de la traduction assermentée. Les trois premiers sont relativement anodins, en commençant par l'invention d'une citation inexistante de Lewis Carroll, l'ajout de propos qu'un auteur (Jose Carlos Somoza) n'as jamais écrits, en passant par des cas de manipulation délibérée dans le doublage de la série télévisée Fawlty Towers et dans le magazine bilingue « Eurodiagnostic », pour aboutir à la distorsion flagrante de la vérité dans la traduction de la Bible. L'auteur soutient que dans l'époque actuelle, appelée par certains celle de la « post-vérité », de pareils cas sont de plus en plus nombreux.

Mots-clés : Post-vérité, complaisance du traducteur, manipulation de la traduction

\section{Abstract}

\section{Translation in the Post-truth Era}

The paper discusses six examples of "indulgence with the truth" in some recent translations in the fields of literature (Lewis Carrol, Jose Carlos Somoza), film (the "Fawlty Towers" series translated into Spanish), mass communication (" Eurodiagnostic »), Bible "inclusive" translation and a case of legal translation. The author aims to show that, perhaps, such translator's inventions, manipulations or distortions of the original texts are currently more frequent in the so called Post-truth era than they used to be in the past.

Key words: Post-truth, translator's indulgence, manipulated translation 